\title{
NUMERICAL SOLUTIONS OF OPTION PRICING MODEL WITH LIQUIDITY RISK
}

\author{
JoNU LEE AND SEKI KIM
}

\begin{abstract}
In this paper, we derive the nonlinear equation for European option pricing containing liquidity risk which can be defined as the inverse of the partial derivative of the underlying asset price with respect to the amount of assets traded in the efficient market. Numerical solutions are obtained by using finite element method and compared with option prices of KOSPI200 Stock Index. These prices computed with liquidity risk are considered more realistic than the prices of Black-Scholes model without liquidity risk.
\end{abstract}

\section{Introduction}

Classical option pricing theory was suggested by Black and Scholes [4] and extended by Merton [7]. They assume that markets are frictionless and competitive. These option pricing models are not applicable in the presence of liquidity risk occurred in we trade underlying assets in illiquid markets.

Liquidity risk is the risk that arises from the difficulty of selling an asset. An investment may sometimes need to be sold quickly. Unfortunately, an insufficient secondary market may prevent the liquidation or limit the funds that can be generated from the asset. Some assets are highly liquid and have low liquidity risk (such as stock), while other assets are highly illiquid and have high liquidity risk (such as a house).

Recently, some investigators have tried to advance option pricing models containing liquidity risk. Amihud and Mendelson [2, 3] suggested that liquidity risk measured by bid-ask spread affects underlying asset prices, less liquid assets give higher expected returns. Karakovsky $[5,6]$ proposed a portfolio for bonds and stocks, and the option pricing equation using the idea of the liquidity number which has a positive sign whenever the underlying asset price goes down or up. Acharya and Pedersen [1] solve explicitly a simple equilibrium model with liquidity risk which a underlying asset's required return depends

Received January 3, 2008.

2000 Mathematics Subject Classification. Primary 65C20, 65M60, 62P05, 91B28.

Key words and phrases. stochastic model, option pricing, liquidity risk, finite element method. 
on its expected liquidity as well as on the covariances of its own return and liquidity with the market return and liquidity.

This paper is organized as follows. In Section 2, we derive an option pricing nonlinear partial differential equation containing liquidity risk. In Section 3, we look for its numerical solution of an European call option price using finite element method with piecewise second-order polynomial basis and test functions. In Section 4, we compare our numerical solutions of the option pricing equation derived in Section 2 with the prices of KOSPI200 stock index option actually traded in the market.

\section{Option hedging portfolio with liquidity risk}

In this section we introduce the modified option pricing model with liquidity risk using the idea of a liquidity number. First of all, we define the market liquidity with connection between the change of the underlying asset price and the notional amount of assets traded in the market.

Definition. The inverse of the partial derivative of the underlying asset price, $S$, with respect to the amount of assets traded in the market, $\widetilde{N}$, is called a market liquidity

$$
L=\frac{1}{\left|\frac{\partial S}{\partial \widetilde{N}}\right|} .
$$

We can also write the market liquidity as

$$
L=\left|\frac{\partial \tilde{N}}{\partial S}\right| .
$$

The market liquidity is always a positive number.

In spite of liquidity being potentially a function of many variables or even stochastic, we will assume that it is a constant parameter as the expected rate of return, $\mu$, and the underlying asset volatility, $\sigma$, are assumed constants in Black-Scholes analysis. This constraint is not necessary, but it will help us to present the framework without additional technical complications. The assumption of constant liquidity by itself should be reasonable for a wide range of market prices. We can find the market liquidity from the change of the amount of underlying assets we trade and the change of the asset price in the market data, we apply $L$ to the underlying asset model.

To derive the option pricing nonlinear partial differential equation containing liquidity risk, we assume several conditions from (H.1) to (H. 7$)$.

(H.1) The underlying asset follows the modified Wiener process

$$
d S=\mu S d t+\sigma S \phi \sqrt{d t}+\frac{1}{L} d N
$$


where $\phi$ is drawn from a standardized normal distribution, $\mu$ is the underlying asset price's instantaneous expected return, $\sigma$ is the instantaneous variance of asset price's return and $N$ is the position we hold in the asset.

(H.2) The short selling of securities with full use of proceeds is permitted.

(H.3) There are no transactions costs or taxes. All securities are perfectly divisible.

(H.4) There are no dividends during the life of the derivative.

(H.5) There are no riskless arbitrage opportunities.

(H.6) Security trading is continuous.

(H.7) The risk-free rate of interest $r$ is constant and the same for all maturities.

We assume that the change of $N$ affects the change of the underlying asset price in our portfolio as the change of $\widetilde{N}$ affects the change of the asset price in the market with same ratio. The first two terms in (2) describe the price dynamics without our trading and the last liquidity term reflects the fact that our trading has an effect on the price level as implied by (1). It is important to realize that the liquidity term is different from a regular stochastic term. We will rehedge our position according to the movement in the asset price rather than randomly. Given a market move $d S$, we know that the change in our position will be $d N$.

We start as in the Black-Scholes analysis and construct a portfolio $\Pi$ consisting of derivative products, whose total value is $V$, and a number $\Delta$, which will be determined later, of shares

$$
\Pi=V-\Delta S,
$$

where $\Delta$ is the total notional amount of the asset. Using Itô's lemma,

$$
d V=\frac{\partial V}{\partial S} d S+\frac{\partial V}{\partial t} d t+\frac{1}{2} \frac{\partial^{2} V}{\partial S^{2}} d S^{2}
$$

we can find the change in value of this portfolio, $d \Pi$, by expanding $V$ about $(S, t)$, as

$$
d \Pi=\left(\frac{\partial V}{\partial S}-\Delta\right) d S+\frac{\partial V}{\partial t} d t+\frac{1}{2} \frac{\partial^{2} V}{\partial S^{2}} d S^{2} .
$$

To eliminate the risk of equation (3) associated with the stochastic movements or adopt a strategy of delta hedging, we choose the number of shares as

$$
\Delta=\Delta(S, t)=\frac{\partial V}{\partial S}(S, t)
$$

We know that the notional $N$ will be equal to $\Delta$ because our portfolio will always be hedged.

Now we can find the option pricing nonlinear partial differential equation with liquidity risk as proving the following theorem. 
Theorem 2.1. If the underlying asset $S$ satisfies

$$
d S=\mu S d t+\sigma S \phi \sqrt{d t}+\frac{1}{L} d N
$$

then the value of the option satisfies the following nonlinear partial differential equation

$$
\frac{\partial V}{\partial t}+r S \frac{\partial V}{\partial S}+\frac{1}{2} \frac{\sigma^{2} S^{2} L^{2} \Gamma}{(L-\Gamma)^{2}}=r V
$$

where $\Gamma$ is the option's gamma $\partial^{2} V / \partial S^{2}$.

Proof. From the equations (2) and (4), we know that

$$
\begin{aligned}
d S & =\mu S d t+\sigma S \phi \sqrt{d t}+\frac{1}{L} d \Delta, \\
d \Delta & =\frac{\partial \Delta}{\partial S} d S+O(d t) .
\end{aligned}
$$

Therefore

$$
d S=\frac{\sigma S L}{L-\Gamma} \phi \sqrt{d t}+O(d t)
$$

We have to find the expression of $d S^{2}$ in the equation (3), it can be easily obtained from the equation (6)

$$
d S^{2}=\frac{\sigma^{2} S^{2} L^{2}}{(L-\Gamma)^{2}} \phi^{2} d t .
$$

$d S^{2}$ is random variable because it contains the random variable $\phi$, but its expected value is

$$
\begin{aligned}
E\left[d S^{2}\right] & =\frac{\sigma^{2} S^{2} L^{2}}{(L-\Gamma)^{2}} E\left[\phi^{2}\right] d t \\
& =\frac{\sigma^{2} S^{2} L^{2}}{(L-\Gamma)^{2}} d t
\end{aligned}
$$

and its variance is zero, so $d S^{2}$ is no more stochastic variable and it has the following value

$$
d S^{2}=\frac{\sigma^{2} S^{2} L^{2}}{(L-\Gamma)^{2}} d t .
$$

From the assumption on no arbitrage opportunities (H.5),

$$
\begin{aligned}
E[d \Pi] & =r \Pi d t \\
& =r\left(V-\frac{\partial V}{\partial S} S\right) d t
\end{aligned}
$$


and the equation (7), we know that the change of the portfolio, (3), becomes

$$
\begin{aligned}
E[d \Pi] & =\left(\frac{\partial V}{\partial t}+\frac{1}{2} \frac{\sigma^{2} S^{2} L^{2} \Gamma}{(L-\Gamma)^{2}}\right) d t \\
& =r\left(V-S \frac{\partial V}{\partial S}\right) d t
\end{aligned}
$$

Consequently, we have

$$
\frac{\partial V}{\partial t}+r S \frac{\partial V}{\partial S}+\frac{1}{2} \frac{\sigma^{2} S^{2} L^{2} \Gamma}{(L-\Gamma)^{2}}=r V
$$

If market is perfectly liquid then the liquidity number $L$ tends to infinity and we obtain the following Black-Scholes equation

$$
\frac{\partial V}{\partial t}+r S \frac{\partial V}{\partial S}+\frac{1}{2} \sigma^{2} S^{2} \frac{\partial^{2} V}{\partial S^{2}}=r V
$$

\section{Numerical solutions for European option pricing}

We try to find numerical solutions of European call option price $V$ satisfied the equation (5). Suppose the stock price $S$ moves from 0 to $a$ ( $a$ can be a sufficiently large value) and $T$ is the time to maturity of the option, then we obtain the mixed boundary conditions of the equation (5) as following,

$$
\left\{\begin{aligned}
V(S, T) & =\max (S-X, 0) \\
V(0, t) & =0 \\
\frac{\partial V}{\partial S}(a, t) & =1
\end{aligned}\right.
$$

where $X$ is the strike price. The difference in time is used to represent the differential term of time on the nonlinear partial differential equation (5), then the equation (5) becomes

$$
\begin{aligned}
& \frac{V\left(S, t_{m}\right)-V\left(S, t_{m-1}\right)}{\Delta t}+r S V^{\prime}\left(S, t_{m-1}\right) \\
& +\frac{1}{2} \frac{\sigma^{2} S^{2} L^{2} V^{\prime \prime}\left(S, t_{m-1}\right)}{\left(L-V^{\prime \prime}\left(S, t_{m-1}\right)\right)^{2}}-r V\left(S, t_{m-1}\right)=0
\end{aligned}
$$

where

$$
\begin{aligned}
\frac{\partial V(S, t)}{\partial S} & =V^{\prime}(S, t) \\
\frac{\partial^{2} V(S, t)}{\partial S^{2}} & =V^{\prime \prime}(S, t)
\end{aligned}
$$


and $\Delta t=t_{m}-t_{m-1}\left(0=t_{0}<t_{1}<\cdots<t_{M-1}<t_{M}=T\right)$. To simplify the notation of above equation, use two following notations

$$
\begin{aligned}
& u(S)=V\left(S, t_{m-1}\right) \\
& \bar{u}(S)=V\left(S, t_{m}\right),
\end{aligned}
$$

and then the equation (10) has the following form

$$
\left(\frac{1}{\Delta t}+r\right) u(S)-r S u^{\prime}(S)-\frac{1}{2} \frac{\sigma^{2} S^{2} L^{2} u^{\prime \prime}(S)}{\left(L-u^{\prime \prime}(S)\right)^{2}}-\frac{1}{\Delta t} \bar{u}(S)=0 .
$$

Since $t$ is not a variable from now on, $u$ is the function with only variable $S$. Let $C_{c}[0, a]$ be the class of continuous functions having the compact support on $[0, a]$. Define

$$
\Phi=\left\{\Psi(S) \in C_{c}[0, a]: \Psi(S) \text { is second-order differentiable }\right\},
$$

we multiply the function $\Psi(S)(\in \Phi)$ to both sides of the above equation and integrate from 0 to $a$,

$$
\begin{aligned}
& \left(\frac{1}{\Delta t}+r\right) \int_{0}^{a} u(S) \Psi(S) d S-r \int_{0}^{a} S u^{\prime}(S) \Psi(S) d S \\
& -\frac{1}{2} \sigma^{2} L^{2} \int_{0}^{a} S^{2} \frac{u^{\prime \prime}(S)}{\left(L-u^{\prime \prime}(S)\right)^{2}} \Psi(S) d S-\frac{1}{\Delta t} \int_{0}^{a} \bar{u}(S) \Psi(S) d S=0 .
\end{aligned}
$$

Because the equation (12) is zero for all test functions $\Psi(S)$, equations (11) and (12) have the same solution and we can obtain the numerical solutions of equation (5) by fining the numerical solutions of (12).

Now we choose admissible basis functions and test functions $\Psi_{i}(S)$ as following three cases.

Case I. $i$ is even

$$
\Psi_{i}(S)=\left\{\begin{array}{ccc}
0 & \text { if } \quad S<(i-2) h \\
\frac{1}{2 h^{2}}(S-(i-2) h)(S-(i-1) h) & \text { if } \quad(i-2) h \leq S<i h \\
\frac{1}{2 h^{2}}(S-(i+1) h)(S-(i+2) h) & \text { if } \quad i h \leq S<(i+2) h \\
0 & \text { if } \quad(i+2) h \leq S,
\end{array}\right.
$$

where $h=S_{i}-S_{i-1}\left(0=S_{0}<S_{1}<\cdots<S_{N-1}<S_{N}=a\right)$ and $N$ is even number.

Case II. $i$ is odd

$$
\Psi_{i}(S)=\left\{\begin{array}{cll}
0 & \text { if } \quad & S<(i-1) h \\
-\frac{1}{h^{2}}(S-(i-1) h)(S-(i+1) h) & \text { if } \quad & (i-1) h \leq S<(i+1) h \\
0 & \text { if } \quad(i+1) h \leq S .
\end{array}\right.
$$


Case III. $i=N$

(15)

$$
\Psi_{N}(S)=\left\{\begin{array}{cl}
0 & \text { if } \quad S<(N-2) h \\
\frac{1}{2 h^{2}}(S-(N-2) h)(S-(N-1) h) & \text { if } \quad(N-2) h \leq S \leq N h .
\end{array}\right.
$$

Suppose that the approximate solution of $u(S)$ is $u_{h}(S)$,

$$
u(S) \simeq u_{h}(S)=\sum_{j=1}^{N} \alpha_{j}^{m-1} \Psi_{j}(S)
$$

since we know the solution at $j=0$ from the second boundary condition. $\alpha_{j}^{m-1}$ is the numerical solution of the equation $(5)$ when $(m-1)$ th time step and $j$ th node point of stock price. Now we perform each integration of the equation (12). Each term can be calculated by three cases that the node point is even, odd and $N$. We can find the first integration as following,

$$
\begin{aligned}
& \int_{0}^{a} u(S) \Psi_{i}(S) d S \\
= & \sum_{j=1}^{N} \alpha_{j}^{m-1} \int_{0}^{a} \Psi_{j}(S) \Psi_{i}(S) d S .
\end{aligned}
$$

Case I. $i$ is even

$$
=\frac{h}{15}\left(-\alpha_{i-2}^{m-1}+2 \alpha_{i-1}^{m-1}+8 \alpha_{i}^{m-1}+2 \alpha_{i+1}^{m-1}-\alpha_{i+2}^{m-1}\right) .
$$

Case II. $i$ is odd

$$
=\frac{2 h}{15}\left(\alpha_{i-1}^{m-1}+8 \alpha_{i}^{m-1}+\alpha_{i+1}^{m-1}\right)
$$

Case III. $i$ is $N$

$$
=\frac{h}{15}\left(-\alpha_{N-2}^{m-1}+2 \alpha_{N-1}^{m-1}+4 \alpha_{N}^{m-1}\right) .
$$

The next term is

$$
\int_{0}^{a} S u^{\prime}(S) \Psi_{i}(S) d S
$$

Case I. $i$ is even

$$
\begin{aligned}
= & \frac{h}{30}\left\{(-4+5 i) \alpha_{i-2}^{m-1}-4(-2+5 i) \alpha_{i-1}^{m-1}\right. \\
& \left.-8 \alpha_{i}^{m-1}+4(2+5 i) \alpha_{i+1}^{m-1}-(4+5 i) \alpha_{i+2}^{m-1}\right\} .
\end{aligned}
$$

Case II. $i$ is odd

$$
=-\frac{2 h}{15}\left\{(-2+5 i) \alpha_{i-1}^{m-1}+4 \alpha_{i}^{m-1}-(2+5 i) \alpha_{i+1}^{m-1}\right\} .
$$

Case III. $i$ is $N$

$$
=\frac{h}{30}\left\{(-4+5 N) \alpha_{N-2}^{m-1}-4(-2+5 N) \alpha_{N-1}^{m-1}+(-4+15 N) \alpha_{N}^{m-1}\right\} .
$$


Because the third integration contains the nonlinear property of the equation (5) and the third boundary condition of (9), we calculate it very carefully. If we compute the third integration in same way as above, then we miss an opportunity which applies the third boundary condition to the equation (5). It is very difficult to find the numerical solutions of the nonlinear partial differential equation (5) which does not contain the boundary condition, even if we can obtain the numerical solutions, their solutions are unstable.

To solve these problems, we divide the test functions into left hand side and right hand side functions when $i$ is even number, first of all we compute the following term

$$
\frac{1}{\left(L-u^{\prime \prime}(S)\right)^{2}}
$$

when $i$ is even, odd and $N$, treating the term as constant with respect to variable $S$.

To include the third boundary condition, we calculate the remainder part of the third integration as the following way,

$$
\begin{aligned}
& \int_{0}^{a} S^{2} u^{\prime \prime}(S) \Psi_{i}(S) d S \\
= & {\left[u^{\prime}(S) S^{2} \Psi_{i}(S)\right]_{0}^{a}-\sum_{j=1}^{N} \alpha_{j}^{m-1} \int_{0}^{a} \Psi_{j}^{\prime}(S)\left(2 S \Psi_{i}(S)+S^{2} \Psi_{i}^{\prime}(S)\right) d S, }
\end{aligned}
$$

the first term applied integration by parts vanishes except the end node point of $S$.

Using all cases of these, we can find the following results of the third integration containing the third boundary condition of (9) in the equation (12).

$$
\int_{0}^{a} S^{2} \frac{u^{\prime \prime}(S)}{\left(L-u^{\prime \prime}(S)\right)^{2}} \Psi_{i}(S) d S
$$

Case I. $i$ is even

$$
\begin{aligned}
= & -\frac{h^{5}}{30} \frac{\left\{\left(4+5 i^{2}\right) \alpha_{i-2}^{m-1}-8\left(1+5 i^{2}\right) \alpha_{i-1}^{m-1}+\left(4+35 i^{2}\right) \alpha_{i}^{m-1}\right\}}{\left\{h^{2} L-\left(\alpha_{i-2}^{m-1}-2 \alpha_{i-1}^{m-1}+\alpha_{i}^{m-1}\right)\right\}^{2}} \\
- & \frac{h^{5}}{30} \frac{\left\{\left(4+35 i^{2}\right) \alpha_{i}^{m-1}-8\left(1+5 i^{2}\right) \alpha_{i}^{m-1}+\left(4+5 i^{2}\right) \alpha_{i+2}^{m-1}\right\}}{\left\{h^{2} L-\left(\alpha_{i}^{m-1}-2 \alpha_{i+1}^{m-1}+\alpha_{i+2}^{m-1}\right)\right\}^{2}} .
\end{aligned}
$$

Case II. $i$ is odd

$$
=\frac{4 h^{5}}{15}\left(1+5 i^{2}\right) \frac{\alpha_{i-1}^{m-1}-2 \alpha_{i}^{m-1}+\alpha_{i+1}^{m-1}}{\left\{h^{2} L-\left(\alpha_{i-1}^{m-1}-2 \alpha_{i}^{m-1}+\alpha_{i+1}^{m-1}\right)\right\}^{2}} .
$$


Case III. $i$ is $N$

$$
\begin{aligned}
= & \frac{a^{2} h^{4}}{\left\{h^{2} L-\left(\alpha_{N-2}^{m-1}-2 \alpha_{N-1}^{m-1}+\alpha_{N}^{m-1}\right)\right\}^{2}} \\
& -\frac{h^{5}}{30} \frac{\left\{\left(4+5 N^{2}\right) \alpha_{N-2}^{m-1}-8\left(1+5 N^{2}\right) \alpha_{N-1}^{m-1}+\left(4+35 N^{2}\right) \alpha_{N}^{m-1}\right\}}{\left\{h^{2} L-\left(\alpha_{N-2}^{m-1}-2 \alpha_{N-1}^{m-1}+\alpha_{N}^{m-1}\right)\right\}^{2}} .
\end{aligned}
$$

Since the value of the last term of the equation (12) is known from the first boundary condition, we may skip the calculation. From all three cases, we can find the numerical solutions of the equation (12) using an iterative method.

In European put option's case, the boundary conditions are following

$$
\left\{\begin{aligned}
V(S, T) & =\max (X-S, 0) \\
V(0, t) & =X e^{-r(T-t)} \\
V(a, t) & =0
\end{aligned}\right.
$$

We can also find numerical solutions of the put option price of the equation (12) with the similar method as above.

\section{Numerical experiments applied to KOSPI200 stock index option}

In this section we compare numerical solutions of the option pricing nonlinear partial differential equation derived in Section 2 with KOSPI200 stock index option prices traded in Korea. We used the finite element method with piecewise second-order polynomial basis and test functions introduced in Section 3, (13), (14), (15), to find the numerical solutions for European call option prices of option pricing equation in (5). KOSPI200 stock index option prices are represented by "point" and One point contract stands for 100,000 Won. We choose two sets of option data which started on January 13, 2006/April 14, 2006 and ended on April 13, 2006/July 13, 2006, respectively. We use the 91date certificate of deposit(CD) yield for risk-free interest rate and the 90-date historical volatility as the underlying asset's volatility. We obtained that the liquidity number, $L$, is 184, 977, 635 when the option maturity is April 13, 2006 and is 2, 198, 684 when the option maturity is July 13, 2006 from the market data. In Tables, we compare KOSPI200 stock index call option prices with our numerical solutions per one week.

We use several abbreviations in tables for the simplification .

- $S$ : price of the KOSPI200 stock index

- HP : highest price of the KOSPI200 stock index option during a day

- LP : lowest price of the KOSPI200 stock index option during a day

- $\mathrm{CP}$ : closing price of the KOSPI200 stock index option

- NS : numerical solutions we found in Section 3 
Table 1: Option prices with liquidity risk (April 13, 2006)

\begin{tabular}{|c|c|c|c|c|c|c|c|c|}
\hline Date & $S$ & $X$ & $\sigma$ & $r$ & LP & HP & CP & NS \\
\hline \hline $01-13$ & 181.71 & 182.5 & 0.1499 & 0.0417 & 6.70 & 6.70 & 6.70 & 6.07453309 \\
\hline $01-20$ & 170.60 & 170.0 & 0.1734 & 0.0416 & 7.40 & 7.40 & 7.40 & 6.78316408 \\
\hline $01-27$ & 178.64 & 180.0 & 0.1660 & 0.0416 & 4.55 & 5.55 & 5.50 & 5.62002550 \\
\hline $02-03$ & 172.67 & 172.5 & 0.1811 & 0.0421 & 7.05 & 7.05 & 7.05 & 6.15618230 \\
\hline $02-10$ & 172.68 & 172.5 & 0.1834 & 0.0426 & 5.00 & 6.50 & 6.20 & 5.87922186 \\
\hline $02-17$ & 172.40 & 172.5 & 0.1864 & 0.0426 & 4.90 & 6.00 & 5.50 & 5.58898244 \\
\hline $02-24$ & 176.96 & 177.5 & 0.1797 & 0.0426 & 4.45 & 5.45 & 5.00 & 4.85385178 \\
\hline $03-03$ & 171.67 & 172.5 & 0.1884 & 0.0426 & 4.05 & 7.10 & 4.40 & 4.51484244 \\
\hline $03-10$ & 170.37 & 170.0 & 0.1931 & 0.0427 & 3.90 & 5.30 & 4.00 & 4.71514329 \\
\hline $03-17$ & 173.48 & 172.5 & 0.1931 & 0.0427 & 2.87 & 4.45 & 5.15 & 4.60676357 \\
\hline $03-24$ & 170.86 & 170.0 & 0.1968 & 0.0427 & 2.67 & 4.00 & 4.00 & 4.04270624 \\
\hline $03-31$ & 176.21 & 175.0 & 0.1987 & 0.0427 & 1.95 & 3.45 & 3.2 & 3.51167468 \\
\hline $04-07$ & 181.97 & 182.5 & 0.1992 & 0.0429 & 0.96 & 1.35 & 1.31 & 1.78380572 \\
\hline
\end{tabular}

Table 2: Option prices with liquidity risk (July 13, 2006)

\begin{tabular}{|c|c|c|c|c|c|c|c|c|}
\hline Date & $S$ & $X$ & $\sigma$ & $r$ & LP & HP & CP & NS \\
\hline \hline $04-14$ & 185.67 & 185 & 0.1996 & 0.0435 & 7.45 & 7.45 & 7.45 & 8.70507152 \\
\hline $04-21$ & 188.2 & 187.5 & 0.1798 & 0.0436 & 6.8 & 7.25 & 6.8 & 7.75396531 \\
\hline $04-28$ & 184.1 & 182.5 & 0.1812 & 0.0436 & 8.35 & 8.35 & 8.35 & 7.96777193 \\
\hline $05-04$ & 186.98 & 187.5 & 0.1713 & 0.0436 & 6.15 & 6.4 & 6.4 & 6.10899756 \\
\hline $05-12$ & 187.27 & 187.5 & 0.1674 & 0.0436 & 5.1 & 6.3 & 5.85 & 5.91491313 \\
\hline $05-19$ & 177.94 & 180 & 0.1812 & 0.0436 & 3.5 & 4.65 & 4.65 & 4.75890293 \\
\hline $05-26$ & 171.83 & 172.5 & 0.1980 & 0.0436 & 4.15 & 5.75 & 5.5 & 5.18738316 \\
\hline $06-02$ & 169.85 & 170 & 0.1926 & 0.0436 & 3.45 & 5.05 & 4.8 & 4.85750321 \\
\hline $06-09$ & 159.61 & 160 & 0.2101 & 0.0441 & 3.9 & 5.55 & 5.4 & 4.20778482 \\
\hline $06-16$ & 163.05 & 162.5 & 0.2284 & 0.0441 & 3.8 & 4.95 & 4.65 & 4.66860053 \\
\hline $06-23$ & 158.88 & 160 & 0.2289 & 0.0455 & 2.41 & 3.35 & 3.25 & 3.1734087 \\
\hline $06-30$ & 167.45 & 167.5 & 0.2367 & 0.046 & 2.53 & 3.05 & 2.75 & 3.24473459 \\
\hline $07-07$ & 164.7 & 165 & 0.2353 & 0.046 & 1.45 & 2.23 & 2 & 1.94443636 \\
\hline
\end{tabular}

\section{References}

[1] V. Acharya and L. Pedersen, Asset pricing with liquidity risk, Journal of Financial Economics 77 (2005), 375-410.

[2] Y. Amihud and H. Mendelson, Asset pricing and the bid-ask spread, Journal of Financial Economics 17 (1986), 223-249.

[3] _ The effect of beta, bid-ask spread,residual risk, and size on stock returns, Journal of Finance 44 (1989), no. 2, 479-486.

[4] F. Black and M. Scholes, The pricing of options and corporate liabilities, The Journal of Political Economy 81 (1973), 637-654.

[5] A. Krakovsky, Gap risk in credit trading, Risk, March (1999), 65-67.

[6] _ Pricing liquidity into derivatives, Risk, December (1999), 65-67. 
[7] R. Merton, Theory of rational option pricing, Bell Journal of Economics and Management Science 4 (1973), 141-183.

JONU LEE

Department of Mathematics

SUNGKYUNKWAN UNIVERSITY

SUWON 440-746, KOREA

E-mail address: jonulee@skku.edu

SEKI KIM

Department of Mathematics

SUNGKYUNKWAN UNIVERSITY

SuWON 440-746, KorEA

E-mail address: skim@skku.edu 